\title{
PACIENTE EN LA UNIDAD DE CUIDADO INTENSIVO Necesidades de la familia frente a su hospitalización
}

\author{
Margarita Pérez Pérez*, Herlidia Taboada Hernández*
}

\section{Resumen}

Dos enfermeras del Hospital de San José realizaron, entre los meses de Abril de 1998 y Mayo de 1999, un estudio relacionado con las familias de los pacientes ingresados a unidad de cuidado intensivo (UCI) con el propósito de identificar sus necesidades y realizar intervención de enfermería encaminada a disminuir los niveles de angustia y aumentar el grado de satisfacción. Se seleccionaron de manera aleatoria diez familias para entrevista y se realizaron dos entrevistas por familia. Se concluye que la familia necesita información más amplia y oportuna; requiere apoyo del equipo de salud de la UCI. La enfermera debe involucrarse más con la familia y cuidar de ella. Rep Med Cir 2000 Nov; 9(2):26-28.

\section{Introducción}

Desde el momento en que un paciente es recluido en la Unidad de Cuidado Intensivo (UCI.), se afecta la unidad familiar; por tanto la atención del equipo de salud se dirigirá tanto al paciente como a la familia.

Sin embargo, en la práctica poco se aplica, pues la atención se centra en el paciente y a la familia es mínimo el tiempo que se le dedica. "La familia considera el ingreso del paciente a la UCI como un debate de él entre la vida y la muerte"(1).

Teniendo en cuenta las inquietudes manifestadas por la familia y la revisión de algunos estudios adelantados en Estados Unidos y España se realizó el presente estudio en la UCI. del Hospital de San José desde el mes de abril de 1998 hasta mayo de 1999. Para recolectar la información se realizaron entrevistas entre los meses de noviembre de 1998 y enero de 1999, concluyendo el estudio en mayo.

Se pretende con el estudio identificar las necesidades de la familia frente a la hospitalización del paciente en UCI. con el propósito de que los profesionales de enfermería realicen cuidados al respecto y así mejore la calidad de atención, disminuyan los niveles de angustia y aumente el grado de satisfacción del usuario.

\footnotetext{
* Facultad de Enfermería, FUCS, Unidad de Cuidado Intensivo Hospital de San José, Bogotá, Colombia
}

\section{Materiales y métodos Método}

El estado se aborda desde el paradigma cualitativo porque busca identificar necesidades y cuantitativo porque utiliza la estadística descriptivo sobre información cuantificable reportada por los mismos.

\section{Tipo de estudio}

Descriptivo, exploratorio longitudinal, se realizó una búsqueda de las necesidades de la familia en un período de tiempo, estableciendo varios contactos con los sujetos de estudio, que permiten la ratificación de la información o la atención de necesidades no expresados en las primeras entrevistas.

\section{Población}

Todos los miembros de la familia de los pacientes hospitalizados en la Unidad de Cuidado Intensivo del Hospital de San José.

\section{Características de la población}

Personas mayores de 15 años, sexo masculino o femenino, lazos afectivos con el paciente, visitas frecuentes (diario o interdiario) y que acepte participar en la investigación.

\section{Muestra}

Se tomó de manera aleatoria a 10 familias de los pacientes ubicados en habitaciones par que cumplieran las características mencionadas. 


\section{Instrumento}

Se utilizó la entrevista semiestructurada, previa validación y aprobación por expertos. Las entrevistas fueron grabadas, luego tabuladas y analizadas, con base en los objetivos propuestos.

\section{Resultados}

Las dimensiones estudiadas son: Información, visitas, vivencias diarias y apoyo.

\section{Información}

Es un tema de preocupación para muchos autores, al respecto, afirma Shomaker que "mucho tiempo y esfuerzo se centraliza en el paciente y en la respuesta del personal, pero con frecuencia la familia es ignorada. Como el paciente, su familia está tensionada por miedo por el resultado (pronóstico), así como por falta de información o por información conflictiva" (2).

En la UCI del Hospital de San José, las familias expresan que reciben algún tipo de información acerca del estado de salud del paciente, causa de ingreso y procedimientos realizados con un alto grado de satisfacción.

En otros aspectos expresan algún grado de inconformidad, tales como no oportunidad en el informe sobre la Monitoria, identidad del médico o enfermera encargados e información telefónica. En la tabla 1 se muestran estos aspectos indicando cuantas familias afirmaron o negaron recibir información al respecto.

Tabla 1

\begin{tabular}{|lll|}
\hline \multicolumn{3}{|c|}{$\begin{array}{l}\text { INFORMACION RECIBIDA POR LOS FAMILIARES DE } \\
\text { LOS USUARIOS DE LA UCI, DEL HOSPITAL DE SAN JOSE, } \\
\text { NOVIEMBRE, DICIEMBRE DE 1998 }\end{array}$} \\
\hline \multicolumn{2}{|c|}{ SI } & NO \\
ASPECTO & 8 & 2 \\
Recibe información en UCI. & 1 & 9 \\
Recibe información oportuna & 2 & 8 \\
Recibe información telefónica & 8 & 2 \\
¿Conoce causa de ingreso / complicación? & 6 & 4 \\
¿Sabe los procedimientos realizados al paciente? & 6 & 7 \\
¿Sabe quién es el Médico encargado? & 3 & 8 \\
¿Sabe quién es la Enfermera encargada? & 2 & 9 \\
¿Sabía cómo encontraría a su familia? & 1 & \\
\hline
\end{tabular}

\section{Visita}

En cuanto a horario y número de visitantes dicen estar de acuerdo, pero siete de las diez familias manifiestan que el tiempo es corto.

\section{Vivencias diarias}

Aquí se quiso buscar la expresión de las familias sobre sus impresiones, sentimientos o creencias vividas diariamente mientras permanece el paciente en cuidado intensivo, bien sea durante su permanencia en la sala de espera o dentro de la unidad.

Los hallazgos más comunes fueron:

"Todos saben para qué es traído un paciente a la unidad de cuidado intensivo".

De las diez familias, ocho manifestaron impresionarse al entrar por primera vez a la UCI. por ver tantos equipo y la forma como encuentran al paciente, refiriéndose a la monitoría, tubos de drenaje, edema y que esto los hace sentir miedo, angustia o ansiedad. Fueron comunes las expresiones: "No la reconocía, me impresionó verla transformada y con todos esos aparatos" "Miedo de ver tantas máquinas y cables" "uno entra y le puede dar un infarto".

Los sentimientos expresados por la familia mientras esperan la hora de visita fueron: "Angustia e incertidumbre por no saber que está pasando con el paciente", "Deseo que abran rápido la puerta", "Temor que avisen que se complicó" e "Impotencia por no poderlo ver o que el paciente necesite algo y no haya quien se lo alcance"

\section{Apoyo}

La familia manifiesta que el tiempo dedicado por los Médicos y Enfermeras para atender sus inquietudes es corto y que es más fácil contactar a la Enfermera que al Médico, pero expresan la necesidad de contar con una persona que tenga más contacto con ellos, lo escuche, los tranquilice y que sea para ello una fuente de apoyo.

\section{Discusión}

La familia del paciente en UCI, está sometida a estrés debido a esta situación, novedosa para la mayoría de ellos. Esto implica que requieran más información tanto en cantidad como en frecuencia y apoyo que cualquier otra familia de pacientes menos complejos.

Es sabido que el tiempo disponible de los profesionales en una unidad es limitado por las múltiples actividades que se deben desarrollar y esto genera cierta confrontación en el sentido que la Enfermera se plantearía: "La familia necesita atención", ¿pero a qué hora lo hago?

$\mathrm{Si}$ bien es cierto que existe esta limitante, vale la pena intentarlo y quizá no sea mucho el tiempo requerido pero si la calidad humana y la disposición para mejorar éste as- 
pecto que va a repercutir para mejoramiento del servicio, la salud familiar y satisfacción del usuario. Además, la interacción con la familia puede ser de gran ayuda para plantear el tratamiento médico y las intervenciones de enfermería. Al respecto, afirma Simpson "las familias guían y median la resolución de problemas, son una fuente de servicio práctico y concreto ayudando durante una crisis en la cual los individuos tienen poca energía personal" (3).

\section{Conclusiones}

La familia necesita información amplia y oportuna del estado del paciente, no sólo referente al diagnóstico, procedimiento de la monitoría, explicarles en términos sencillos y entendibles el pronóstico, los resultados de algunos exámenes o cirugías.

La familia necesita el apoyo de los miembros del equipo de salud, que puedan escucharlos, comunicarse con frecuencia y orientarlos en diversos aspectos.

La Enfermera debe tener en cuenta a la familia para su plan de trabajo.

\section{Referencias}

1. García A, y otros. Comunicación e información a los familiares en la unidad de cuidados intensivos. Enfermería Clínica. 1995; 5 (3) Mayo-Junio;100.

2. Shomaker TH. Tratado de medicina crítica y terapia intensiva. Panamericana;1985:1289.

3. Simpson T. La familia omo una fuente de apoyo par el adulto críticamente enfermo. AACN. 1991; 2 (Mayo):

\section{Bibliografia}

Alspach W. Cuidado Intensivo en el Adulto. $2^{\mathrm{a}}$ edición. Interamericana. 1987.

Amaya de PP. Enfermería familiar: Aspectos conceptuales, metodológicos de la práctica familiar. Memorias $\mathrm{V}$ congreso nacional de humanización en salud. Bogotá 1995.

Charles FK. Ayuda a la familia del paciente ingresado en una UCI. Nursing, nov 1991.

Cray L. Un proyecto de colaboración: iniciación de un programa de intervención familiar en una Unidad Médica de Cuidados.

Dugas BW. Tratado de enfermería práctica. $4^{\mathrm{a}}$ edición. Interamericana. México. 1987.

Gotzone MT. Análisis de la problemática en la relación enfermera -enfermo en la UCI..Revista Roll de enfermería.1993. Kozier B, y otros. Conceptos y temas en la práctica de enfermería. $2^{a}$ edición. Interamericana. 1995.

Labiano TJ. problemática de los familiares de pacientes ingresados en la UCI. febrero 1987.

Lopez FL. Critical Care family need inventory: a cognitive research utilization approach. Critical Care nurse. Agosto 1995.

Soy MT. Cuidados Intensivos. Manuales prácticos de Enfermería. McGraw - Hill. Bogotá 1997.

Ubeda BI. Revista Roll de Enfermería: Atención a la familia : ¿utopía o realidad?. Agosto 1995.

Warren N. Perceived needs the family members in the Critical care Nursing. Nov. 1993.

Whittaker JY. Psicología. $4^{\circ}$ edición. Interamericana. México. 1986.

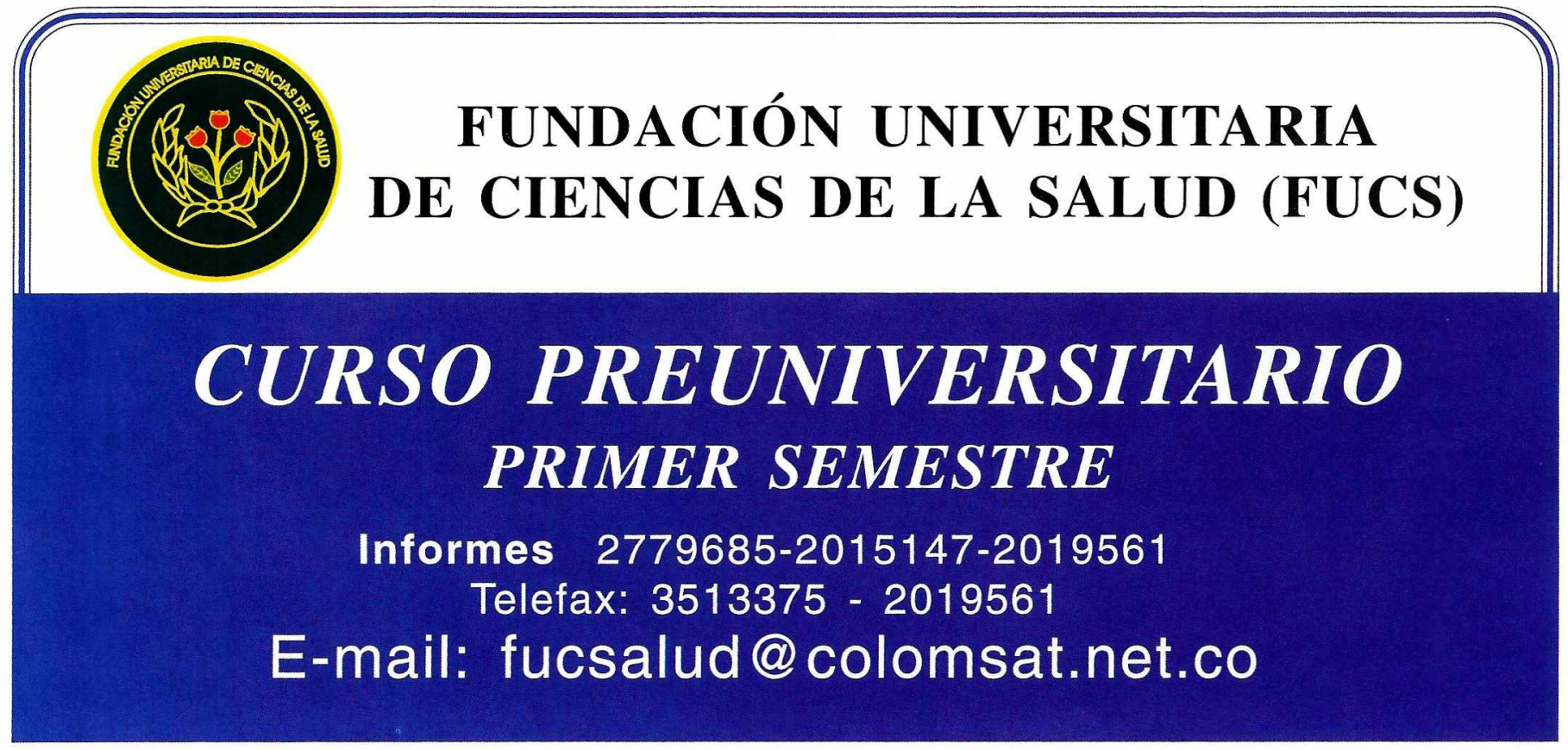

